"100 años de radio, 96 de radio universitaria"

Lucía Casajús, Noelia Giorgi

Question/Cuestión, Vol 2 N66, Agosto 2020.

ISSN: $1669-6581$

https://perio.unlp.edu.ar/ojs/index.php/question/

IICom - FPyCS - UNLP

\section{“100 AÑOS DE RADIO, 96 DE RADIO UNIVERSITARIA"}

\author{
"100 YEARS OF RADIO, 96 OF UNIVERSITY RADIO"
}

\section{Lucía Casajús}

Doctora en Comunicación y Magíster en Periodismo Digital y Multimedia

(Universidad Jaume I, España). Lic. en Periodismo y Comunicación Social (UNLP). Responsable de articulación institucional y académica en la Dirección de Medios UNDAV. Autora del libro sobre divulgación científica en la radio universitaria Lo dijo la radio. Entonces habrá que investigar. Ha sido docente de grado y posgrado en la UNLP y en la UNDAV. https://orcid.org/0000-0002-4477-4580

\section{Noelia Giorgi}

Profesora universitaria (UNLP) y maestranda en Comunicación y Creación Cultural por la Fundación Walter Benjamin. Responsable de programación de 
Radio UNDAV y producción general en la Dirección de Medios de la Universidad Nacional de Avellaneda, donde también es responsable de la articulación de contenidos en redes universitarias y realizadora de coproducciones junto a las emisoras Concepto Radial del Instituto Tecnológico de Monterrey (México), Onda Campus de la Universidad de Extremadura (España) y las emisoras que integran la red de Radio Internacional Universitaria (RIU). Autora del libro sobre divulgación científica en la radio universitaria Lo dijo la radio. Entonces habrá que investigar. Es docente de grado en la Universidad Nacional de Avellaneda. https://orcid.org/0000-0002-6455-8607

\section{Resumen}

A menos de cuatro años del nacimiento de la radio, el 27 de agosto de 1920, nace también en Argentina la radio universitaria, el 5 de abril de 1924. Contemporánea a la Reforma Universitaria de 1918, y como consecuencia de ella según sus documentos fundadores, se inauguraba un modelo de radio concebido con vocación extensionista, ligado a la educación, la ciencia y la cultura.

\section{Palabras clave}

Radio, radio universitaria, investigación

\section{Abstract}

Less than four years after the birth of radio, on August 27, 1920, university radio was also born in Argentina, on April 5, 1924. Contemporary to the University Reform of 1918 , and as a consequence of it, according to its founding 
documents, a radio model conceived with an outreach vocation, linked to education, science and culture, was inaugurated.

\section{Keywords}

Radio, university radio, research

\section{La radio cumple 100 años y las radios universitarias son parte de la historia}

LR11 Radio Universidad Nacional de La Plata es considerada la primera emisora radiofónica universitaria del mundo, y su nacimiento marcó el inicio de un centenario camino en la creación de emisoras universitarias en distintos países de América Latina, y puso a la Argentina a la vanguardia en la creación de radios en las universidades.

La radio universitaria es una realidad desde hace 96 años y se trata de un fenómeno cada vez más extendido del que forman parte estudiantes, docentes, investigadorxs, trabajadorxs y autoridades universitarias, así como también distintos actores sociales. Un gran conglomerado de estaciones que tienen lugar en todo el mundo y que, a pesar de esta cuestión, es un sistema de medios no del todo conocido socialmente.

\section{Radio universitaria en Argentina}

Fue en el mes de noviembre de 1923 cuando el entonces presidente de la Universidad Nacional de La Plata, Benito Nazar Anchorena, presentó en el Consejo Superior el proyecto de creación de una emisora universitaria para completar la obra de extensión con el fin de vincular a la universidad con el medio social en el que se encuentra (Antonucci et al., 2009: 16). Así, el 5 de 
abril de 1924, junto con la apertura formal del ciclo lectivo, se inauguraba oficialmente LR11 Radio Universidad Nacional de La Plata, y nacía la radio universitaria como un medio de comunicación estrechamente vinculado a la educación y a la investigación, concebido como medio cultural ligado a la sociedad, tal como lo manifestara en el discurso de inauguración de la emisora el entonces rector:

A la Universidad de La Plata le corresponde la iniciativa de haber empleado una estación radiotelefónica no sólo como excelente elemento de enseñanza e investigación para la Radiotécnica sino también para fines de divulgación científica, o sea, como elemento de extensión universitaria (...). De tal modo, al par que desarrolla una obra completa de difusión cultural, sirve para vincular más aún la Universidad con el medio social en que actúa, devolviendo con ventaja al país el esfuerzo que la Nación realiza para sostenerla (Fragmento del discurso de Benito Nazar Anchorena. Web de Radio Universidad de La Plata. Consulta: 12/12/2012).

La primera radio universitaria del mundo, planteaba como uno de sus postulados el de "vincular la Universidad al pueblo, que debía inspirar la tarea llamada de extramuros o de Extensión Universitaria" (Vázquez, 2012: 54).

Siete años después, se crea la segunda emisora universitaria argentina en la Universidad Nacional del Litoral (UNL) que, el 18 de agosto de 1931, pone al aire LT10 con el objetivo de "tender un puente entre la Universidad y la Comunidad" (Levatti, 2016: 91).

Desde ese momento, y en el marco de las autonomías universitarias, fueron puestas en el aire una importante cantidad de emisoras universitarias, sobre todo a partir de la restauración de la democracia en 1983, hasta alcanzar un efecto de múltiples instalaciones tras la sanción de la Ley 26.522 de Servicios 
de Comunicación Audiovisual (LSCA), en el año 2009. Tal como señala Tedesco (2013) "La sanción de la Ley de Servicios de Comunicación Audiovisual significó el reconocimiento del derecho pleno de las universidades nacionales a la radiodifusión, permitiéndoles saltar las barreras legales que les imponía el viejo decreto 22.285". Así, como apunta Giorgi:

El peso y contundencia de la LSCA en los medios universitarios argentinos quedan evidenciados en la multiplicación de emisoras universitarias, cuya virtual duplicación en número sobre las existentes previas a la ley, vino acompañada por decisiones fundamentales por parte de las autoridades nacionales que advirtieron la necesidad de fomentar no solo la aparición de más emisoras de radio y señales de TV, sino también su asistencia financiera y mecanismos de capacitación. Un proceso que va a quedar interrumpido y definitivamente clausurado al asumir el nuevo gobierno y el cambio de paradigma que se inicia en diciembre de 2015 (Giorgi, 2016: 5).

Más de 30 universidades públicas, entre las que se hallan las 16 creadas entre los años 2009 y 2015, se sumaron a la lista de casas de altos estudios con radio, hasta alcanzar en conjunto el número de 62 estaciones de radio en la actualidad.

Hoy, la radio universitaria argentina está cerca del centenario. En este recorrido, algunas universidades han avanzado en la creación de más de una emisora, bajo el impulso de su presencia en la extensión territorial. Este es el caso, por ejemplo, de la Universidad Nacional de Entre Ríos (UNER) y su Sistema Integrado de Radios (SIRUNER) con tres emisoras: la cabecera en la ciudad de Paraná (Radio UNER Paraná), creada en 2011, y la radicación de otras dos radios en Concepción del Uruguay y Concordia.

Las radios de las universidades públicas argentinas son mayoritariamente 
estaciones de frecuencia modulada y en el caso de las nacidas en amplitud modulada, como ocurrió en el sector privado hasta la renovación de las concesiones, cuentan con su radio FM. Un ejemplo es Radio Universidad de La Plata que puso en marcha su FM en el año 1989 durante el auge de proliferación de las FM a finales de la década del 80 en el contexto de la vuelta a la democracia. La mayoría de las radios universitarias cuentan, también, con emisión online y existen incluso algunas que solo tienen emisión online en streaming o bajo el sistema podcast tales como RadioPerio, de la Facultad de Periodismo y Comunicación Social de la UNLP, o Radio Conurbana, de la Facultad de Ciencias Sociales de la Universidad Nacional de Lomas de Zamora (UNLZ).

\section{La radio universitaria, de Argentina al mundo}

La puesta en marcha de la radio de la Universidad Nacional de La Plata marca el inicio de un extenso camino en la aparición de emisoras universitarias que comenzaron a desarrollarse en distintos países de América Latina "siendo Colombia, México y Chile, los países que continuaron la tendencia con el surgimiento de la Emisora Cultural Universidad de Antioquia (1933), Radio Universidad Nacional Autónoma de México (1937) y Radio Universidad Técnica Federico Santa María (1937)" (Casajús, 2015: 130).

Actualmente, también hay radios universitarias en Uruguay, Honduras, Cuba, Ecuador, Costa Rica, Nicaragua, Bolivia, Venezuela, Brasil, Perú y Paraguay. Latinoamérica es una región con una gran trayectoria en materia de radio universitaria. Una cuestión reconocida a nivel mundial que, incluso, ha hecho que sea tomada como modelo a seguir por países como España, que se adentró en la emisión universitaria en 1987 con el nacimiento de la radio de la 
Universidad de La Laguna'. Como señalan Ixs investigadorxs españoles Marta y Martín-Pena (2014) "La experiencia de Latinoamérica en lo referente a la emisión universitaria es un caso paradigmático".

En la actualidad, a 96 años de su nacimiento, la radio universitaria es un fenómeno que tiene lugar en todo el mundo. Existe también un gran desarrollo de radios universitarias en Norteamérica, las College Radios; y en otros países de Europa como Italia, Francia o Portugal; e incluso se registran experiencias en Oceanía, Asia y África (Vázquez, 2012: 94-95).

\section{El trabajo en redes de radios universitarias}

Un factor importante en el reconocimiento y trascendencia internacional de la radio universitaria es, sin dudas, el trabajo en red de las emisoras. En los últimos años, son varias las acciones que se han venido implementando por parte de miembros y gestorxs de estas radios, así como de asociaciones que las agrupan, para hacer visible el fenómeno de la radio universitaria.

En los países latinoamericanos existen redes que reúnen a las radios de las universidades tanto para potenciar sus iniciativas individuales, como para revalorizar el trabajo conjunto y compartir su caudal educativo, científico y cultural. Tal es el caso de Argentina, donde se conformó en 1998 la Asociación de Radiodifusoras Universitarias Nacionales Argentinas (ARUNA); de México, con la Red de Radios Universitarias de México (RRUM, antes llamada SINPRIES); de Chile, con la Red de Radioemisoras Universitarias de Chile (REUCH); de Colombia con la Red de Radio Universitaria de Colombia (RRUC); y de Ecuador, con la Red de Radios Universitarias del Ecuador (RUE). A partir de estas integraciones por países, las emisoras de la región conformaron en 2009, la Red de Radio Universitaria de Latinoamérica y el 
Caribe (RRULAC) que agrupó a unas 300 emisoras universitarias de nueve países y a la que en 2015 se integró también España a través de la Asociación de Radios Universitarias de España (ARU). A pesar de sus variados perfiles, las radios universitarias encontraron en la red un espacio concebido con el espíritu de la construcción colectiva desde el que se impulsaron numerosas actividades "con el afán de expandir la idea de una comunicación plural y democrática" (RRULAC, 2016)ii. En el año 2017, RRULAC pasa a transformarse en una red internacional para albergar a emisoras de otros países interesadas en formar parte de la Red. Así, durante el IV Encuentro de la RRULAC, realizado en España, se incorporaron nuevas redes y radios de distintos países que motivaron la necesidad de replantear el nombre de la RRULAC y sus estatutos para dar paso a la constitución de Radio Internacional Universitaria (RIU), red de redes presidida por la Universidad de Extremadura (España), y con la vicepresidencia institucional a cargo de la Universidad Nacional de Avellaneda (Argentina). Actualmente, a cuatro años de su creación, la RIU está integrada por unas 500 emisoras universitarias. Aglutina tanto a redes de radios universitarias como ARUNA (Argentina); RRUE (Ecuador); RRUM (México); REUCH (Chile); RadUni (Italia); RUBRA (Brasil), además de radios asociadas de Uruguay, Paraguay, Brasil, Costa Rica, Nicaragua, Portugal e Italia. También cuenta con la adhesión de Spanish Public Radio, una emisora multimedia radicada en Chicago (EEUU) que tiene la misión de transmitir y apoyar contenido radiofónico a la comunidad de habla hispana residente en Estados Unidos; y de Radio ILCE la emisora del Instituto Latinoamericano de Comunicación Educativa, con sede en México. 


\section{Perfiles e identidades de la radio universitaria}

La identidad de una radio universitaria está definida por sus particularidades y características. Es decir que si bien entre las emisoras existen diversidades según modos de organización y funcionamiento, programación, cuestiones presupuestarias o de financiamiento, ubicación geográfica y sentido territorial, recursos técnicos, políticas universitarias o trayectorias, sí que pueden establecerse algunos rasgos comunes.

Estas radios se enmarcan en el contexto universitario, funcionan con el respaldo de las universidades a las que pertenecen y son parte del sistema de medios universitarios, y a la vez se insertan en la sociedad y son también conformantes del sistema de medios de comunicación social y de la radiofonía. Tienen, por una parte, una clara función de divulgación del conocimiento que se genera en las casas de estudios y de visibilización de las actividades y actores universitarios, así como un fuerte compromiso con la educación, la investigación y el aprendizaje y, por otra parte, una función informativa y de servicio público con compromiso social. Como señalan Aguaded y Martín (2013: 64) "Las emisoras universitarias, en general, se han postulado como radios que traspasan la frontera de lo exclusivamente instructivo $\mathrm{y} / \mathrm{o}$ formativo para trascender a un carácter social y de servicio ciudadano". Son radios que trabajan desde la universidad hacia y con la sociedad, entendiendo que el vínculo de la universidad con su entorno y su territorio es fundamental, y lo es también la relación y el compromiso de sus medios de comunicación con el ámbito social en el que se encuentran. Como señalan Dido y Barberis:

La gestación y creación de una radio universitaria ofrecen una excelente oportunidad para habilitar una vía de expresión y difusión de planes, inquietudes, proyectos de una comunidad. Como ninguna otra, posee las 
condiciones apropiadas para convertirse en un foro de debate de aquellos asuntos que inciden en diferentes grupos comunitarios y para actuar como factor dinámico de la comunidad de pertenencia (Dido y Barberis, 2006:15).

El perfil de las radios universitarias se delinea en gran parte a partir de su programación, que configura el espíritu de estas emisoras. En este sentido, mayoritariamente, cuentan con una programación integrada por contenidos alternativos a los emitidos por las radios comerciales. Sus propuestas programáticas, con lugar para diversas voces y temáticas, marcan la impronta de estas radios. Unas emisoras con una programación variada en la que convergen los contenidos universitarios y del entorno social. Como señalan Aguaded y Contreras (2011: 5), estas radios apuestan por "Abrir espacios de comunicación donde se expresan las inquietudes y el quehacer diario de las universidades, desde el punto de vista científico, cultural o de extensión a la sociedad". Álvarez Villa y Ramírez Queralt (2005) también se refieren a esta cuestión y consideran que, "Estos medios se plantean como iniciativas para la aproximación de la actividad universitaria a la sociedad y como canales de comunicación en el seno de la misma Universidad".

Asimismo, la divulgación científica en la programación de las emisoras universitarias es una cuestión inherente a su esencia como medio de comunicación que, inmerso en el ámbito universitario de formación y generación de conocimiento científico, debe bregar por la extensión de estos conocimientos hacia la sociedad, de una manera accesible. En este sentido, la radio como medio de comunicación presenta interesantes características para albergar contenidos de divulgación científica: 
La radiodifusión es un canal idóneo para difundir democráticamente la ciencia. Se trata de un medio barato, al alcance de la mayoría, lo que lo convierte en el más popular. Es sencillo en la producción de sus contenidos, claro en su registro hablado, y veloz, inmediato y directo en su difusión (Gómez, 2002: $62)$.

Una cuestión mencionada también por Segura Anaya, que se refiere a ella vinculándola específicamente al ámbito de la radio universitaria:

La práctica universitaria contempla como fundamentos de su actividad: la docencia, la investigación y la transferencia de conocimiento y en este ámbito, la radio se convierte en un medio especialmente interesante para la divulgación de la ciencia y de las investigaciones que se generan en el seno de la Universidad (Segura Anaya, 2014: 45).

Como explica Martín Pena, la divulgación científica:

Debe jugar un papel principal en las propuestas programáticas de las emisoras, es decir, debe ser uno de los objetivos capitales de las estaciones universitarias, dando cumplimiento a una de las funciones básicas de las instituciones académicas, la labor de divulgación del conocimiento entre la ciudadanía (Martín Pena, 2013: 538).

Contreras y Parejo (2013: 35-36) consideran que las radios universitarias "son las herramientas perfectas, e idóneas, para mantener ese constante diálogo con la sociedad a la que a través de la divulgación se transfiere el conocimiento generado en sus laboratorios" y deben ser entendidas "como claves para cumplir los objetivos de extensión a la ciudadanía".

De esta manera, las radios universitarias promueven en su programación espacios vinculados a la actualidad universitaria, a la divulgación científica y a la difusión de contenidos de sus carreras, y al mismo tiempo abren espacios a 
la participación de los miembros de la comunidad universitaria: docentes, no docentes, estudiantes, investigadorxs y autoridades.

Además, muchas de estas radios también tienen entre sus objetivos apoyar la formación de los estudiantes de carreras relacionadas con el periodismo y la comunicación, cumpliendo con la labor de enseñanza de la universidad y convirtiéndose en medios que dan lugar a la experimentación. La radio universitaria tiene un rol fundamental como espacio de aprendizaje y laboratorio de experimentación y permite a los estudiantes desempeñar las prácticas periodísticas en un medio de comunicación durante la carrera dentro del propio espacio universitario y en el marco de los programas curriculares. Para esto, la articulación académica del medio universitario con las carreras es el eje central a partir del cual implementar los proyectos de participación de los estudiantes.

\section{La investigación en radio universitaria}

La investigación en materia de radio universitaria es relativamente reciente si la comparamos con otras materias de estudio en el ámbito de la radio. En la última década, tanto en Latinoamérica como en España, las publicaciones de estudios e investigaciones sobre la radio universitaria han crecido de la mano de diversos autores y, en muchos casos, en gran parte a partir del trabajo en red de estas emisoras a través de las asociaciones que las agrupan y desde las que se han impulsado eventos científicos, encuentros, libros y textos específicos para abordar y reflexionar en torno a este fenómeno. De esta manera, el corpus teórico y la documentación de proyectos, experiencias y prácticas de la radio universitaria viene creciendo a partir de aportaciones de académicos e investigadores así como de trabajadoras y trabajadores de estos medios, a través de artículos, ponencias, libros, tesis y otras publicaciones que 
estudian la radio universitaria y que dan cuenta de las características y experiencias de estos medios de comunicación.

En el libro Lo dijo la radio, editado por las autoras de este artículo y publicado en 2017, Lucía Casajús y Daniel Martín Pena, dedican un capítulo al estado de investigación en materia de radio universitaria y hacen un recorrido por las diferentes publicaciones y autores de América Latina y España que trabajan esta temática, desde la década del 90 . En este sentido, señalan que:

El experto en Comunicación y Educación Daniel Prieto Castillo fue uno de los primeros en pensar y analizar el escenario de la radio universitaria en 1996 mediante una ponencia en el I Festival Centroamericano de la Radio de Costa Rica, titulada "Las emisoras Universitarias frente a las transformaciones de fin de siglo" (Casajús y Martín Pena, 2017: 83).

En el ámbito argentino, entre los años 2000 y 2010, encontramos ya los primeros artículos publicados en revistas científicas:

En 2001, el profesor Gustavo lovino, junto a otros autores, publica el artículo "La oferta comunicativa de las radios universitarias del NOA" en la revista de la SeCyT de Santiago del Estero. En 2007, Carolina Asuaga, profesora de la Universidad de La República (Uruguay) publica su estudio "Gestión de las radios universitarias: Definiendo la Estrategia". En 2010, los profesores de Comunicación Social, Carlos Milito y Lucía Casajús publican los principales resultados de la investigación realizada desde la Cátedra Taller de Producción Radiofónica I de la Facultad de Periodismo de la Universidad Nacional de La Plata titulada "Las Radios Universitarias argentinas en Internet: Relevamiento, desarrollos, modelos y enfoques" (Casajús y Martín Pena, 2017: 83-84).

En cuanto a las primeras producciones bibliográficas en nuestro país, los profesores de Comunicación de la Universidad Nacional de La Matanza 
(UNLaM), Juan Carlos Dido y Sergio Barberis publican en 2006 el libro Radios Universitarias: Principios, funciones, objetivos; en 2009, los profesores de la UNLP Sergio Antonucci, María Elena Beneitez, Sandra Gabay y Omar Turconi editan Radio Universidad Nacional de La Plata: 85 aniversario: 1924-2009: toda una vida; y en 2015, Mario Giorgi, de la Universidad Nacional de Avellaneda (UNDAV), publica el libro La radio universitaria. Hacia la consolidación de una Red Autónoma de Medios; y Oscar Bossetti, de la Universidad Nacional de Quilmes (UNQ) y la Universidad de Buenos Aires (UBA), y Ricardo Haye, de la Universidad Nacional del Comahue (UNComa), editan el libro Radios universitarias argentinas. Una red pública y federal para ejercer el derecho a la comunicación que compila artículos de diversxs autorxs e inicia con esta temática la serie editorial La Radio del Nuevo Siglo.

Como hemos mencionado, Casajús y Martín Pena (2017) en el artículo citado detallan Ixs investigadorxs, autoras y autores que han trabajado el tema tanto en Argentina como en el contexto iberoamericano, y allí puede consultarse los trabajos, artículos científicos, ponencias y tesis doctorales realizadas en España y en América Latina y el Caribe, incluso en coautorías entre investigadorxs de distintos países. Pero es interesante recopilar aquí una serie de producciones colectivas que integran trabajos acerca de la radio universitaria en distintos países. La primera de ellas, es la publicación presentada en 2011 en el acto oficial de la conformación de la Asociación de Radios Universitarias de España (ARU) titulada La radio universitaria como servicio público para una ciudadanía democrática. Un libro colectivo fruto del Curso de Verano de la Universidad Internacional de Andalucía (UIA), organizado junto a la Universidad de Huelva (UHU), y celebrado en 2010 bajo el título Ondas de las Universidades: las radios universitarias como servicios 
ciudadanos de Comunicación. La publicación fue coordinada por José Ignacio Aguaded y Paloma Contreras (UHU). Un año después se presenta un segundo libro con la participación de autores de distintos países, titulado Las radios universitarias, más allá de la radio. Las TIC como recursos de interacción radiofónica, coordinado por Cinta Espino (UHU) y Daniel Martín Pena (Universidad de Extremadura - UEx). En 2013 se edita la tercera publicación coordinada por Paloma Contreras (UHU) y Macarena Parejo (UEx), bajo el título Más Ciencia. Cómo trabajar la divulgación científica desde las radios universitarias. En 2014, surge un nuevo libro titulado Radio Universitaria en América y Europa, coordinado por Daniel Martín Pena (UEx) y Miguel Ángel Ortiz (Universidad Complutense de Madrid - UCM). En 2016, se publican los libros: Perspectivas y prospectivas de la radio universitaria en la era digital coordinado por Daniel Martín Pena (UEx), Carmen Marta (Universidad de Zaragoza - UNIZAR) y Miguel Ángel Ortiz (UCM), fruto de la participación en el VII Congreso Internacional Latina de Comunicación Social celebrado en la Universidad de La Laguna (ULL); y Encrucijadas del nuevo milenio. Radio, Comunicación y Nuevas Tecnologías, compilado por Ricardo Haye y Oscar Bosseti, que recopila algunas de las ponencias presentadas en las $X$ Jornadas La Radio del Nuevo Siglo, celebradas en la Universidad Nacional de Avellaneda (UNDAV), la mayoría de las cuales se dedica a analizar distintos aspectos de la radio universitaria de la mano de profesorxs, investigadorxs y miembros de estas emisoras. En 2018, la Radio Internacional Universitaria publica Radios Universitarias en Marcha. Hacia la construcción de una contra agenda mediática, editado y coordinado por Daniel Martín Pena y Agustín Vivas Moreno, de la Universidad de Extremadura, fruto del IV Encuentro RRULAC en que se conformó la RIU. Y en 2019, bajo la coordinación de Eliana Albuquerque 
y Norma Meireles, desde el Centro de Comunicación, Turismo y Artes (CCTA) de la Universidade Federal da Paraíba (UFPB), se edita el libro colectivo Rádios Universitárias experiências e perspectivas.

También cabe destacar la realización de monográficos sobre radio universitaria en revistas científicas tales como el de la Revista EdMetic de la Universidad de Córdoba, España (vol. 2, núm. 2, 2013) bajo el título Antecedentes, estado de la cuestión y prospectiva de la radio universitaria; o el de la Revista Mediterránea de la Universidad de Alicante (Vol. 9, Núm. 1, 2018) Las radios universitarias en la convergencia digital.

Para finalizar, cabe señalar que como indican Casajús y Martín Pena (2017) es "a partir de 2011, cuando se registra un fuerte aumento de las contribuciones al estudio de este tema" lo que ha permitido "conocer más de cerca la realidad de las emisoras universitarias iberoamericanas, y reflexionar sobre ella, gracias a publicaciones que han abordado temas muy variados: radio universitaria como democratizadora de la comunicación; las Tecnologías de la Información y la Comunicación en las emisoras universitarias; las grillas de programación y sus contenidos, con especial interés en la divulgación de la ciencia; la historia del medio en América Latina y Europa o las modalidades de gestión y organización de estas estaciones" y ha supuesto "una impronta importante para la investigación del medio radiofónico universitario con el surgimiento de artículos, comunicaciones en congresos o libros específicos sobre el fenómeno. Este hecho, se debe en gran parte a la creación de asociaciones, redes o agrupaciones de emisoras tanto a uno como otro lado del Atlántico".

\section{A modo de conclusión}


Las radios universitarias son parte de la historia de la radio, con 96 años de recorrido desde la inauguración de la primera emisora de este tipo y el surgimiento y desarrollo de centenares de nuevas radios universitarias en todo el mundo a lo largo de casi un siglo.

La radio universitaria se propuso desde su nacimiento ser la voz de las universidades, una voz comprometida con su entorno social y cultural asumiendo la responsabilidad como medio de comunicación que visibiliza la labor de las instituciones académicas y abre el espacio a la reflexión crítica a partir de la participación de múltiples actores.

Es imprescindible valorizar y visibilizar la importancia del rol de la radio universitaria no solo como herramienta de comunicación, sino también como un espacio que conjuga un fuerte compromiso con la educación y el aprendizaje, una función de servicio público con responsabilidad periodística y compromiso social; para informar desde una comunicación inclusiva, democrática y alternativa, integrando las voces de la comunidad universitaria, de los actores políticos y del territorio, y extenderla a la sociedad en general.

En este sentido, tanto el trabajo en redes de emisoras universitarias como la investigación y producción bibliográfica en esta temática, es una cuestión central a la que, desde la Dirección de Medios de la Universidad Nacional de Avellaneda, hemos querido contribuir mediante este artículo.

\section{Bibliografía}

- Aguaded, J.I. y Contreras, P. (2011) (Coords.). La radio universitaria como servicio público para una ciudadanía democrática. A Coruña: Netbiblo.

- Aguaded, J.I. y Martín, D. (2013). Educomunicación y radios 
universitarias: panorama internacional y perspectivas futuras. Chasqui, 124; 63-70.

- Álvarez Villa, A. y Ramírez Queralt, A. (2005): Los objetivos de las emisoras de radio universitarias a través de su programación. En II Congreso Iberoamericano de Comunicación Universitaria, Granada. Consultado el 2/02/2013 en http://dialogo.ugr.es/cicu2005/com/com36.pdf

- Antonucci, S. Beneítez, M.E.; Gabay, S. y Turconi, O. (2009) Radio Universidad Nacional de La Plata 85 Aniversario 1924-2009. Toda una vida. La Plata, EDULP.

- Casajús, L. (2015). Radios universitarias y redes sociales. Tesis Doctoral. Universitat Jaume I.

- Casajús, L. y Martin Pena, D. (2017). La investigación en materia de radio universitaria en Latinoamérica y España. En Casajús, L. y Giorgi, N., Lo dijo la radio. Entonces habrá que investigar. (pp. 81-98). UNDAV Ediciones, Avellaneda.

- Contreras, P. y Parejo, M. (Coord.) (2013). +Ciencia. Cómo trabajar la divulgación científica desde las Radios Universitarias. Salamanca: Comunicación Social.

- Dido, J. y Barberis S. (2006). Radios universitarias. Principios, funciones, objetivos. Universidad Nacional de La Matanza. Bs. As. Argentina.

- Giorgi, M. et al. (2016): "Radio UNDAV. La experiencia de creación y puesta en marcha de la radio de la Universidad Nacional de Avellaneda". Actas de Periodismo y Comunicación. Revista electrónica sobre congresos, encuentros y jornadas de la Facultad de Periodismo y Comunicación Social. ISSN 2469-0910 
https://perio.unlp.edu.ar/ojs/index.php/\%20actas/article/view/3737

- Gómez, O. (2002) La divulgación científica en el medio radiofónico: algunos apuntes. En Mediatika, 8, 59-68. Consultado el 3/01/2014 http://www.euskomedia.org/PDFAnlt/mediatika/08/08059068.pdf

- Levatti, A. (2016). Desafíos y encrucijadas de la radiofonía universitaria. En O. Bosetti y R. Haye (Eds.), Encrucijadas del nuevo milenio: radio, comunicación y nuevas tecnologías (pp. 89-110). Avellaneda, Argentina: UNDAV Ediciones - ARUNA.

- Marta, C.; y Martín-Pena, D. (2014): "Antecedentes, estado de la cuestión y prospectiva de las Radios Universitarias". EDMETIC, Revista de Educación Mediática y TIC .3 (1), 1-6

- Martín Pena, D. (2013). Radios universitarias en España: plataformas de comunicación interactiva y redes de colaboración. Tesis doctoral. Universidad de Huelva.

- Segura Anaya, A. (2014). Divulgación científica en las radios universitarias españolas. En Edmetic, 3; 44-60.

- Tedesco, M. (18/09/2013). Políticas de comunicación y radio universitarias. Página/12. Disponible en https://goo.gl/47R6Pz

- Vázquez Guerrero, M. (2012). La radio universitaria en México y España. Estudio de la participación y formación de los jóvenes. Tesis. Universidad Pompeu Fabra.

\section{Notas}


' El primer caso de experiencia radiofónica universitaria en España lo encontramos en Radio UNED, la emisora de la Universidad Nacional de Educación a Distancia, que nace en 1974, aunque se trata de un caso particular con un modelo centrado exclusivamente en transmisiones formativas. ii Web de la RRULAC http://rrulac.org/ Fecha de consulta: 14/12/16 